\title{
Künstliche Intelligenz, Roboter, digitalisierte Vernetzung - Epochenwechsel in der gesellschaftlichen Organisation der Arbeit und Grundeinkommen
}

\section{Paul Kellermann}

Zusammenfassung: Auf der Basis von Künstlicher Intelligenz, Robotern und digitalisierter Vernetzung ließe sich nach den physisch oder finanziell erzwungenen Arbeitsverhältnissen in Sklaven-, Lohnarbeitsund Geldgesellschaften eine neue gesellschaftliche Organisation der Arbeit für ein wirtschaftlich gesichertes, freieres Arbeitssystem mit Einkommensgarantie entwickeln. Rational-funktionale Argumente für garantiertes Einkommen sind zum einen: Wenn privatwirtschaftlich in Automaten investiert wird, um auf Märkten deren Produkte absetzen zu können, muss die gewünschte Nachfrage mit Geld ausgestattet sein. Nachfrage kann nur dann entstehen, wenn beides, Bedarf und Geldverfügbarkeit, gegeben sind. Zum anderen: Arbeitszwang ist, gleichgültig in welcher Gesellschaft und auf welchen Gebieten, wirtschaftlich weniger effizient als intrinsisch motivierte Tätigkeit.

Schlüsselwörter: Gesellschaftliche Organisation der Arbeit, Künstliche Intelligenz, Roboter, Grundeinkommen

\section{Artificial Intelligence, Robots, Digital Networking - Shift of the Epochs in the Social Organization of Work and Basic Income}

\begin{abstract}
Based on artificial intelligence, robotics, and digitized networking, an economically secure, freer labor system with guaranteed income could be developed. In fact, the physically respectively financially forced labor conditions in slave, wage work and money societies are outdated. Rational functional arguments for a new social organization of work with guaranteed income are: If money is privately invested in machines, the intent is to sell the products. That can be realized only in the case that demand exists. But
\end{abstract}

P. Kellermann $\square$

Alpen Adria-Universität, Institut für Soziologie Universitätsstraße 65, 9020 Klagenfurt, Österreich

E-Mail: Paul.Kellermann@aau.ac.at 
demand can occur only when both, needs and availability of money, are given. In addition: forced labor is, in whatever society and in whatever areas, economically less efficient than intrinsically motivated activity.

Keywords: Social Organization of Work, Artificial Intelligence, Robots, Basic Income

„Die Schwierigkeit liegt nicht so sehr in den neuen Gedanken, als in der Befreiung von den alten, die sich bei allen, die so erzogen wurden, wie die meisten von uns, bis in die letzten Winkel ihrer Geistesart verzweigen.“ (John Maynard Keynes, 1883-1946)

„Ich fürchte den Tag, an dem die Technologie unsere Menschlichkeit überholt. Die Welt wird dann eine Generation von Idioten sein.“ (Albert Einstein, 1879-1955)

„Robotics and artificial intelligence are beginning to fundamentally change the relative profitability and productivity of investments in capital versus human labor, creating technological unemployment at all levels of the workforce, from the North to the developing world." (Hughes 2014, p. 45)

„Manchmal scheint es, als schaffte sich der Mensch gerade ab. Als übergäbe er die Kontrolle an Computer, intelligente Maschinen und soziale Netzwerke. Als wäre seine Zukunft bloß noch die einer Randexistenz auf einem Planeten, der von Algorithmen organisiert und beherrscht wird. Als wäre die Gattung Mensch bald nur noch der Störfaktor in einer Welt, die ohne ihn besser auskäme.“ (Brauck et al. 2015, S. 9)

„Jibo kann hören, sehen, sprechen, fotografieren, und er lernt ständig dazu. Er erinnert an Verabredungen, lässt sich E-Mails diktieren, spielt mit den Kindern, bestellt Essen von einem Lieferservice.“"(a.a.O., S. 13)

\section{Vorbemerkung}

Die Diskussion um Folgen des beschleunigten Einsatzes neuer technischer Entwicklungen (Künstliche Intelligenz, digitale Kommunikation, Roboter ...) beschränkt sich zumeist auf die Veränderungen im Erwerbsarbeitssystem und in seinem Arbeitsmarkt, obwohl die Folgen viel weiter reichen. Zudem ist auch die Diskussion um Arbeit beschränkt, weil wesentliche Bereiche menschlicher Arbeit nicht berücksichtigt werden. Allgemein konzentriert sich die Besorgnis auf ,technological unemployment“, auf die ,job economy“, also auf abhängige Beschäftigung. Übersehen wird dabei, dass gesellschaftlich erforderliche Arbeit in vielen Bereichen entweder auf andere Weise oder auch gar nicht erledigt wird.

1 Weitere Zitate zur Einstimmung in die Problematik sind im Anhang zu finden. 
Die in verschiedenen Studien vorausgesagte Ersetzung von bis zur Hälfte der aktuell beschäftigten Arbeitskräfte in den nächsten zwei Jahrzehnten durch neuartige Roboter (Automaten, Künstliche Intelligenz) (z.B. Frey, Osborne 2013) wird unter zwei, derzeit vorherrschen Gesichtspunkten diskutiert:

In Ländern mit Bevölkerungszuwachs und vergleichsweise jungem Durchschnittsalter wie in den USA wird ein Anstieg der Erwerbslosigkeit durch den stark zunehmenden Robotereinsatz befürchtet. Allerdings sollte man annehmen, dass angesichts der vielen ungelösten Weltprobleme von globaler Armut und stark gewachsener Erdbevölkerung über weltweite Naturzerstörung und Epidemien bis hin zu den unzähligen ethnischen, religiösen, wirtschaftlichen und politischen Konflikten in den verschiedenen Erdregionen genügend zu tun wäre. Dass dem nicht entsprochen wird, liegt wohl an der fehlgeleiteten Organisation gesellschaftlich erforderlicher Arbeit: Was im gesellschaftlichen Interesse bearbeitet werden sollte, woran aber nichts zu verdienen ist, wird vernachlässigt.

In Ländern mit einer rückläufigen Anzahl an Geburten und steigender Anzahl aus dem Erwerbsleben ausgeschiedener Personen wird durch Robotereinsatz ein Ausgleich zwischen erforderlichen und verfügbaren Arbeitskräften erhofft. Unter Berücksichtigung der stark gewachsenen Erdbevölkerung, von etwas über einer Milliarde Menschen zu Beginn des 20. Jahrhunderts zu sechs Milliarden an seinem Ende und der Prognose weiteren starken Anstiegs in den kommenden Jahrzehnten ${ }^{2}$, sollte aber eigentlich kein Mangel an Arbeitskräften bestehen, da alle Menschen bis auf die Hilfsbedürftigen über qualifizierbares Arbeitsvermögen verfügen. Auch hier fehlt es an angemessener Organisation der Arbeit infolge des allgemeinen Unverständnisses einer der wesentlichsten Funktionen von Geld als ein modern-funktionales Instrument der Vermittlung von menschlichem Bedarf und menschlichem Potential durch Arbeit.

In beiden Denkansätzen wird anscheinend völlig außer Acht gelassen, dass wir Menschen ein Amalgam, eine „Melange“, von natürlichen, sozialen und kognitiven Komponenten sind. Diese nicht auflösbare Zusammensetzung des entwickelten menschlichen Lebens, jeder menschlichen Persönlichkeit, jeweils nur unter einem verengten Menschen- und Weltbild statt in ihrem Potential wahrzunehmen, kann nicht zur Sicherung oder Verbesserung der allgemeinen Lebensbedin-

2 Im Verhältnis zu dem größten Weltproblem, nämlich gegenüber dem Wachstum der Erdbevölkerung, sind „Job economy“, Auto-Abgasskandal, Präsidentenwahlen, Wirtschaftswachstum, Börsencrashs, Überalterung und andere Probleme weniger bedeutsam. Sie wurden großteils durch starkes Bevölkerungswachstum verursacht, mit den Folgen überregionaler Kriege, Hungersnöte und darauffolgenden Massenfluchtbewegungen. Es hat Jahrhunderte, ja Jahrtausende gedauert bis zu Beginn des 20. Jahrhunderts rund 1.25 Milliarden Menschen die Erde bevölkerten. Um diese Anzahl zu erreichen, hat es gerade einmal die ersten 16 Jahre des 21. Jahrhunderts gebraucht, dann war bereits die Anzahl von über sieben Milliarden erreicht und das Wachstum geht weiter. Angesichts dieser Problemdimension sind Anstrengungen wie Studien zum „Teilchenbeschleuniger“ bei Genf, zur virtuellen „Unsterblichkeit“" von Menschen, zur Erforschung des Mars, aber auch die im Hinblick auf die Folgen nicht durchdachte Weiterentwicklung von „künstlicher Intelligenz“ Ausdruck von Unvernunft - schlimmer: Blindheit gegenüber den existentiell drohenden Katastrophen aufgrund der Entwicklung der Erdbevölkerung. Verbesserung der allgemeinen Lebensverhältnisse scheint das einzig erfolgreiche Mittel zur Verringerung jenes Wachstums zu sein (vgl. Sobotka et al. 2015). 
gungen führen. Beide demographischen Problemlagen erfordern eine andere als die bisherige gesellschaftliche Organisation der benötigten Arbeit.

So müsste ein grundsätzlich anderer Denkansatz über die vorherrschenden Sichtweisen auf Arbeitskräfte und Arbeitsplätze hinausreichen. Ein solcher Ansatz sollte nach Möglichkeiten suchen, Probleme und deren Bearbeitung in wesentlicheren Zusammenhängen zu sehen. Insbesondere müsste er die Möglichkeit verfolgen, den von alters her gegebenen Zwang zur Erarbeitung der erforderlichen Mittel des Lebens zu mildern, also freie Tätigkeit auf der Basis einer gesicherten Existenz zu gestatten. Einen Ansatz in diese Richtung zu skizzieren, soll im Folgenden versucht werden.

Bei raschem und massivem Einsatz von Robotern und anderen elektronischen Innovationen müssten sich freilich alle drei Sichtweisen den Fragen nach den in Zukunft noch bestehenden Rahmenbedingungen stellen: Wie werden sich das Verhalten der Menschen und der Umgang miteinander in der durch Automaten geprägten Lebenswelt verändern? Wird es weiterhin Gegensätze und Konflikte zwischen arm und reich in den einzelnen Ländern und zwischen ihnen geben; zwischen ethnischen und religiös begründeten Gruppierungen? Werden die tradierten Aufgaben und Ziele der Regierungen sowie der gesellschaftlichen Institutionen beispielsweise der Familie, Gerichte, Parteien, Kirchen, Gewerkschaften, Universitäten, Konzerne noch die gleichen sein? Und grundsätzlich: Welcher Gesellschaftstyp wird herrschen? Weiterhin der Globale Konkurrenzkapitalismus? Welche Rolle spielt dann Geld?

Widerspricht die extrem gesteigerte Abhängigkeit der Menschen vom fehlerlosen Funktionieren der Automaten nicht prinzipiell der bisherigen unternehmerischen und individuellen Freiheitslehre („Neoliberalismus“)? So ist auch zu problematisieren, inwieweit ein rascher und vermehrter Einsatz jeder Art von ,artificial intelligence" auf Akzeptanz bei Unternehmen und in Privathaushalten trifft: Unternehmen müssten ihre „Philosophie“ anpassen und entsprechend grundlegend neu investieren, was nur erfolgt, wenn der Einsatz Gewinn erwarten lässt. Und Privathaushalte müssten über die erforderliche Kaufkraft der von Robotern erzeugten, beziehungsweise angebotenen Leistungen verfügen, aber auch mental mit den Neuerungen zurechtkommen.

\section{Arbeit, Wirtschaftsleistungen und Geld}

Um zu verstehen, was die Besetzung bisheriger (und auch künftiger) Arbeitsplätze durch Automaten bedeutet, muss zuerst verstanden werden, was „Arbeit“ bedeutet: Menschliche Arbeit ist letztlich eine Tätigkeit, die auf die Bereitstellung von Mitteln zur Stillung von Bedürfnissen gerichtet ist. Letzter „Maßstab“ ist ihr Beitrag zur Sicherung und Verbesserung der gesellschaftlichen Lebensverhältnisse (Kellermann 2014 a, S. 6). Mit dieser Norm ist gesagt, dass verantwortliche menschliche Arbeit auch bei massivem Einsatz von Automaten unabdingbar ist. 
Die zur gesellschaftlichen Reproduktion, Produktion und Innovation zu erbringende Arbeit ist in den Industrieländern de facto sehr differenziert: zunächst wird bezahlt und unbezahlt, in Freien Berufen oder auch ehrenamtlich Arbeit geleistet; dann im Interesse der Privathaushalte, der Organisationen und im Interesse des Allgemeinwohls.

In keiner Weise bearbeitet werden Tausende Probleme, wie sie seit 1972 von der Union of International Associations in ihrer fortgeführten Veröffentlichung „,The Encyclopedia of World Problems and Human Potential" aufgelistet werden. Kurz: Das öffentliche Denken beschränkt sich aktuell auf ein mental und geschichtlich begrenztes System der Arbeitsorganisation nach dem Modell der Erwerbs- - noch beschränkter - der Lohnarbeit.

Dieses anachronistisch gewordene, häufig als zwanghaft empfundene System ließe sich überwinden, indem der Zusammenhang von allgemeinem Bedarf, kollektivem Leistungsvermögen und ausgleichender Geldverfügbarkeit erkannt und funktional gestaltet würde: Die technisch erreichte höhere Produktivität des Arbeitsvermögens und das erweiterte Leistungsangebot durch automatisierte Prozesse kann marktlogisch nur genutzt werden, wenn Nachfrage durch Bedarf und Geldverfügbarkeit gesichert ist: Fehlt eines von beidem, Bedarf oder Geld, gibt es keine Nachfrage.

Nachfrageausfall ist häufig der Grund für die unbefriedigende wirtschaftliche Lage in vielen Ländern, weil zwar von den Zentralbanken (in Japan, USA, Europa) große Geldmengen zur Verfügung gestellt werden, diese aber hauptsächlich dorthin gelangen, wo kaum Bedarf an realen Wirtschaftsleistungen besteht:

„Seit Beginn der Interventionen im Jahr 2009 ist das US-BIP um magere 7,9 Prozent gewachsen, der Aktienindex S\&P 500 aber um 215 Prozent.“ (Urschitz 2015, S. 15)

Zugleich ist offensichtlich, dass hoher Bedarf an Arbeitsleistungen zur Milderung der „Weltprobleme“ besteht. Würden die Geldmengen ,intelligent“ zur Organisation der gesellschaftlich erforderlichen Arbeit verwendet, könnten bestehende Probleme durch das ja verfügbare menschliche und betriebliche Produktionsvermögen gemildert werden. Doch die gedankliche Fixierung auf Geld, Einkommen und Profit verhindert die Erkenntnis und den Einsatz von Geld als funktionalem Mittel der politischen Arbeitsorganisation im gesamtgesellschaftlichen Interesse.

Völlig verkannt wird offensichtlich im beschränkten Gelddenken ${ }^{3}$ die Tatsache, dass Geld nur dann realen Wert hat, wenn für dieses Geld erwünschte Waren zu kaufen sind. Und Waren entstehen durch die Nutzung von Produktionsmitteln. Nur die verfügbaren Wirtschaftsleistungen sind folglich die Wertsicherung (die Deckung) des Geldes (Kellermann 2014 a, S. 32). Dementsprechend sind die Erarbeitung von Waren und deren Absatz die entscheidenden Größen

3 „Beim Denken an Geld endet das Denken“ - ein Aspekt des „Moneyismus“(Kellermann 2007, S. 115 ff.). 
für das nach dem Bruttoinlandsprodukt $t^{4}$ berechnete Wirtschaftswachstum sowie für den Grad

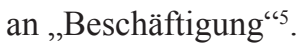

\section{Technology Assessment}

Der Einsatz von neuen Produktionsmöglichkeiten hat immer Folgen für die gesellschaftliche Organisation der Arbeit. Die Erfindungen von Rad, Pflug und Uhr haben vergleichsweise langsam die Arbeitsorganisation verändert. Aber immer kürzer wurden die Phasen der Neuerungen nach Beginn des Buchdrucks, der mechanischen, der elektrischen, automatischen und zuletzt der digitalen Produktionsmöglichkeiten.

Nach wissenschaftlichen Analysen betreffen digitale Innovationen etwa die Hälfte der zu Beginn des 21. Jahrhunderts bestehenden Arbeitsplätze für Menschen im Laufe seiner ersten Jahrzehnte $^{6}$. Allerdings hat der steigende Einsatz von elektronischen Hilfsmitteln (,Automaten") Auswirkungen auf weit mehr Themen und Probleme:

- Information und Wissen wachsen stetig und erweitern die Datenmengen; zugleich überlagern sie Denken und Verstehen.

- Erweiterte Funktionen der Automaten übernehmen bisherige menschliche Alltagstätigkeiten, verstärken damit die Abhängigkeit der Menschen von ihnen.

- Zunehmende Vernetzung erhöht die Komplexität des Systems, was sowohl Effizienzsteigerungen als auch größere Risiken bedeuten kann.

4 „Das Bruttoinlandsprodukt (BIP) stellt das umfassendste Maß für die Gesamtproduktion einer Volkswirtschaft an Waren und Dienstleistungen dar. Es ist die Summe der Geldwerte des Konsums (C), der Bruttoinvestitionen (I), der Staatsausgaben für Waren und Dienstleistungen (G) und der Nettoexporte (X), die in einer Volkswirtschaft innerhalb eines Jahres hergestellt werden.“ (Samuelson, Nordhaus 2005, S. 604) Kürzer: „Das nominelle Bruttoinlandsprodukt (BIP) entspricht der Gesamtsumme der Bruttoausgaben der Endverbraucher für inländische Leistungen zum Kaufpreis.“(Kellermann 2014 b, S. 253)

5 Umgekehrt ist immer wieder irreführend zu hören: Das Wirtschaftswachstum reiche nicht aus, um die Zahl der Arbeitslosen zu senken. Ein klassisches Beispiel für vordergründiges Denken - eine Folge wird als Ursache missverstanden.

6 „We examine how susceptible jobs are to computerisation. To assess this, we begin by implementing a novel methodology to estimate the probability of computerisation for 702 detailed occupations. [...] According to our estimates, about 47 percent of total US employment is at risk." (Frey, Osborne 2013) Damit ist allerdings nicht gesagt, dass es insgesamt weniger Arbeitsplätze geben wird. $\mathrm{Zu}$ vermuten ist, dass andersartige Arbeitspositionen entstehen. Welcher Art und welchen Ausmaßes hängt wesentlich von der Art der Finanzierung ab. 
- Das Verhältnis Menschen/Automaten bei der Kontrolle von Prozessen ${ }^{7}$ verändert sich: Was erleichtert das alltägliche Leben der Menschen, was ist von Menschen nicht mehr durchschaubar?

- „Autonome Automaten“ können Entscheidungen übernehmen und Menschen dadurch entlasten, aber in gleichem Maße auch entmündigen.

- „Autonome Waffen “ verändern die Relation der Todesfälle bei „Freund“ und „Feind“.

- Globale Bevölkerungsbewegungen können human und inhuman durch neue Technologien beeinflusst werden.

- Die Herstellung und der Gebrauch von Automaten haben Auswirkungen auf den Klimawandel.

- Durch Künstliche Intelligenz, Roboter und digitale Vernetzung werden Arbeit und Wirtschaft auf eine neue Basis gestellt.

Nur das letzte Thema, der Wandel von Arbeit und Wirtschaft im Rahmen der gesellschaftlichen Entwicklung, soll in folgenden Thesen angesprochen werden. Freilich sind die anderen, nur sehr selten behandelten, Themenkomplexe nicht von geringerer, aber von andersartiger Bedeutung. Sie müssten jeweils in gleicher Weise beobachtet und bearbeitet werden.

\section{Der Wandel von Arbeit und Wirtschaft}

In Beantwortung der qualitativen Frage „Wer erledigt im historischen Wandel der Arbeitsorganisation die gesellschaftlich erforderliche Arbeit?" lassen sich grob drei Modelle unterscheiden:

- Die feudale Gesellschaft Kleinbauern, Handwerker, lokale Händler, Sklaven, Leibeigene, Knechte und Mägde erledigen den Großteil der Alltagsarbeit unter Verwendung wenig entwickelter Hilfsmittel; es besteht eine fast geldlose Herrschaft einer auf Erbe und „Gottesgnadentum“ strukturierten Gesellschaft; Macht und Reichtum werden in Landbesitz und dessen Bevölkerung gesehen. Zur meist körperlichen Arbeit wird physisch gezwungen. - Feudale Gesellschaften waren und sind alle, in denen ein kleiner Teil der Mitglieder über Gebiete und deren Bewohner herrscht, wobei die große Mehrheit die erforderliche Arbeit leistet.

7 „Die Technik kann nur das tun, wofür sie von Menschen programmiert wurde. Und wie man am Flug 1549 gesehen hat (Landung eines voll besetzten Flugzeugs auf dem East River/Manhattan am 15.1.2009, P.K.), lässt sich ein bis dahin undenkbares Ereignis wie ein doppelter Triebwerksausfall nach einem Vogelschlag in geringer Höhe über einer Großstadt nicht planen.“ (Löger 2016, S. 22) 
- Die Lohnarbeitsgesellschaft

Die Lohnarbeitsgesellschaft organisiert die Arbeit auf der Basis von Zeit und Geld, wobei wiederum ein kleiner Teil der Gesellschaftsmitglieder über Reichtum und Macht verfügt, der weitaus größere gezwungen ist, seine Fähigkeiten gegen Lohn anzubieten. Arbeiter und Angestellte in Fabriken und Büros, Dienstleistungen und Banken prägen die Struktur der Gesellschaft. Die mit Geld organisierte betriebliche Arbeitsteilung führt zu Spezialisierungen, Produktivitätssteigerungen und erweiterten Märkten. Die Arbeitsmotivation orientiert sich zunehmend an Gelderwerb, obwohl ein „Recht auf Arbeit“ gefordert wird. ${ }^{8}$ Die Entlohnung der abhängig Beschäftigten erfolgt auf Zeitbasis nach sozialer Differenzierung. Die Industrialisierung erfasst stetig mehr Bereiche der Erwerbsarbeit. Es entstehen der Industrie- und Finanzkapitalismus.

- Die befreite Gesellschaft

Das gerade angebrochene Zeitalter der Künstlichen Intelligenz, der Robotik und digitalen Vernetzung erfordert eine andere, grundsätzlich innovative gesellschaftliche Arbeitsorganisation. Diese Epoche ermöglicht die Weiterentwicklung von zunächst geldloser Sklavenarbeit im Feudalismus über geldbezogene Lohnarbeit im Kapitalismus zur finanziell gesicherten freien Arbeit in einem sozialen Liberalismus.

Die quantitative Veränderung der Erwerbsarbeitsstruktur in der sehr kurzen Zeitspanne 1950 - 2010 in Deutschland bietet ein Beispiel für den Wandel von Erwerbsarbeit und Wirtschaftsstruktur:

Erwerbstätige im Inland nach Wirtschaftssektoren, Deutschland (WZ2008/ GENESIS-Online)

$\begin{array}{ccccc}\text { Jahr } & \text { Insgesamt } & \begin{array}{c}\text { Primärer Sektor } \\ \text { Argrarkultur }\end{array} & \begin{array}{c}\text { Sekundärer Sektor } \\ \text { Industrie }\end{array} & \begin{array}{c}\text { Tertiärer Sektor } \\ \text { Dienste } \\ \%\end{array} \\ 1950 & 1000 & \% & \% & 32,5 \\ 1960 & 19570 & 24,6 & 42,9 & 38,3 \\ 1970 & 26063 & 13,7 & 47,9 & 45,1\end{array}$

8 Ein „Recht auf Arbeit“ wurde bereits 1793 im Rahmen der großen französischen Revolution (ab 1789) verlangt wie ebenso in der Revolution von 1848 und fand dann Eingang in die UNO-Menschenrechtsdeklaration ein Jahrhundert später. Damit konnte damals eigentlich nur das Recht auf Leben in der entstehenden industriellen Lohnarbeitsgesellschaft gemeint sein. Doch fast niemand, damals wie heute, der diese Forderung erhebt, denkt dabei an Arbeit im Sinne des Schaffens von Gebrauchswerten, aber nahezu alle meinen den Erwerb von Geld als erforderliches Tauschmittel zum Kauf der Mittel des Lebens. 


$\begin{array}{lllll}1980 & 27420 & 5,1 & 41,1 & 53,8 \\ 1990 & 30409 & 3,5 & 36,6 & 59,9 \\ 2000 & 39386 & 1,9 & 28,7 & 69,4 \\ 2010 & 40587 & 1,6 & 24,5 & 73,8\end{array}$

Trotz des starken Wandels bleibt das zu Erwerbsarbeit zwingende System die Basis: Nur zur allmählichen Verkürzung der Erwerbsarbeitszeit (Tages-, Wochen-, Jahres- und Lebensarbeitszeit) wurde die Chance, die sich durch steigende Produktivität ergibt, genutzt. Dagegen könnte das Arbeitssystem epochal neu konzipiert werden: statt Erwerbszwang, Arbeitsfreiheit; statt Aversion der Erwerbsarbeit gegenüber (z.B. Flucht von Erwerbsarbeit in freie Zeit wie Wochenende, Urlaub, Pension) intrinsische Arbeitsmotivation. Hindernisse eines grundsätzlichen (,paradigmatischen") Wechsels der Arbeitsorganisation in Betrieben und Gesellschaft sind herrschender Zeitgeist und vorherrschende Menschen-, Gesellschafts- und Weltbilder.

Eine neue gesamtgesellschaftliche Organisation der Arbeit könnte auf den folgenden Komponenten basieren: autonome Produktion durch Menschen und Automaten. Unter ,autonom“ ist dabei Selbstregulierung zu verstehen, die bei den Menschen im Rahmen von persönlichen Motiven und staatlichen Gesetzen, im Fall der Automaten im Rahmen von policies und soft ware erfolgt. Menschliche und maschinelle Abweichungen von den Regeln im Sinne von Störungen sind dabei nicht auszuschließen. Solche Friktionen sind insbesondere aus der grundsätzlichen Inkompatibilität der Charakteristiken von Mensch und Maschine zu erwarten: Menschen sind zugleich natürliche (biotische) und gesellschaftliche (soziale) Wesen; Automaten entstanden aus langwährenden Entwicklungen durch menschlichen Geist und Gebrauch als komplizierte Maschinen.

Die in Generationen entwickelten Produktionsmöglichkeiten ${ }^{9}$ erlaubten im 21. Jahrhundert, eine vom Arbeitszwang weitgehend befreite Gesellschaft und von Herrschaft unabhängige Arbeitsverhältnisse zu entwickeln: Automaten und digitale Technologien erledigen wachsende Anteile der Arbeitstätigkeiten. Autonome Arbeit der Menschen wird in partizipativen Lebens- und Arbeitsgemeinschaften auf Basis eines gesicherten Grundeinkommens sowie freier

9 „Das gegenüber der handwerklichen und mechanischen Produktion wesentliche gesellschaftliche Moment der automatischen Produktion ist die Entlassung der lebendigen menschlichen Arbeitskraft aus der unmittelbaren Gütererzeugung. Abgesehen davon, daß Menschen in den verbliebenen Beständen handwerklicher und mechanischer Produktion beschäftigt sind, beschränkt sich in der automatischen Produktionsform der Bedarf an menschlicher Arbeitskraft auf die Bereiche von Forschung und Entwicklung, auf die Umsetzung der entwickelten Technologie in kapitalisierte Technik (also automatische Produktionsmittel), auf die globale Kontrolle sowie auf die Instandhaltung der Kapitalaggregate. Die Auswirkungen der automatischen Produktionsform erfassen die gesamten sozialen Beziehungen; vor allem betreffen sie die Arbeitsorganisation, die Qualifikation der Arbeitskraft, die Gütererzeugung, die wirtschaftspolitischen Beziehungen auf dem Weltmarkt, die Besitzverhältnisse und die Beschäftigung - sowie von all diesen Bereichen abhängig - die innere gesellschaftliche Verfassung." (Kellermann 1981, S. 39) 
Erwerbsarbeit nach Motiven und Interessen, Kreativität und Spontanität in Selbstorganisation und Selbstverantwortung ermöglicht.

\section{Grundeinkommen und zusätzliche Erwerbsarbeit}

Jede Gesellschaft muss erarbeiten, was sie zur Sicherung und Verbesserung ihrer Lebensbedingungen braucht. Dazu muss sie mit den verfügbaren Ressourcen „ökonomisch“, d.h. wirtschaftlich umgehen. Zugleich haben alle Menschen Bedürfnisse und die meisten verfügen über ein Arbeitsvermögen, um zu schaffen, was gebraucht wird. Dieses Vermögen muss sowohl gesellschaftlich als auch persönlich entwickelt und verwendet werden, um Bestand zu haben. Ergänzende Aspekte der Arbeitsverhältnisse sind:

- anthropologisch: Zumindest Kinder und alle unverbildeten Menschen haben das Bedürfnis, tätig zu sein (,homo faber").

- sozialanthropologisch: Alle Menschen haben das Verlangen, von ihrer Bezugsgruppe (reference group) anerkannt und integriert zu werden/zu bleiben. Dieses Bedürfnis wird durch Beiträge zur Bestandssicherung der Gruppe erreicht. Arbeit/Kooperation ist dafür das wichtigste Moment.

- soziologisch: Die weit fortgeschrittene Arbeitsteilung erfordert einen Austausch der wirtschaftlichen Leistungen (Güter und Dienste). Dieser Austausch wird in der gesellschaftlichen Entwicklung immer ausschließlicher mit Hilfe des Mediums „Geld“ organisiert. Deshalb verlangt das individuelle Leben in einer solchen „Geldgesellschaft“ die persönliche Verfügbarkeit über Geld.

- ordnungspolitisch: Die noch zu Beginn des 21. Jahrhunderts bestehende Industriegesellschaft erhält/erhielt ihre gesellschaftliche Ordnung durch das „Beschäftigungssystem“ beziehungsweise Erwerbsarbeit.

- kognitiv: Denken an Arbeit und darauf zurückgehendes Handeln konzentrieren sich in der entwickelten Geldgesellschaft auf Geld und damit vor allem auf Einkommen durch Erwerbsarbeit.

- global: Um im „Globalen Konkurrenzkapitalismus“ nicht nur Mitläufer oder gar nur „Spielwiese“ für mächtigere Gesellschaften zu sein, muss jede Gesellschaft ihre Ressourcen (vor allem ihr menschliches Arbeitsvermögen) bestmöglich entwickeln.

Beengtes Denken korreliert mit der Unfähigkeit, ein anderes Modell der gesellschaftlichen Organisation von Arbeit als das des historisch entstandenen Beschäftigungssystems auch nur sich vorstellen zu können. Ein Modell für die Imagination von Alternativen, um mit diesen zu experimentieren (s. unten), wäre folgendes: Ein bestimmbarer Anteil von persönlicher Geldver- 
fügbarkeit im Sinne eines garantierten „Grundeinkommens“ ließe sich je nach Bedürfnissen mit einem weiteren Anteil in Form von Erwerbsarbeit kombinieren.

Der Übergang zu einer Gesellschaft, in der jeder über ein Mindestmaß an Geld verfügt, um das eigene Überleben zu sichern, könnte ein sinnvoller Weg sein. Die damit erreichbare selbständige Tätigkeit als anthropologisches Bedürfnis könnte menschliche Potentiale sich entfalten lassen, die das individuelle wie das kollektive Leben zumeist befriedigender gestalten ließen als in der überholten Form der Arbeitsorganisation. Wer sozial-anthropologisch bedingte Bedürfnisse nach Zugehörigkeit, Anerkennung und sinnvoller Tätigkeit empfindet, erbringt brauchbare Leistungen über das gesicherte Geldeinkommen hinaus entweder mit oder ohne Honorierung. Die Organisation des Wirtschaftskreislaufs erfolgt über das Instrument Geld in Form von einander ausgleichenden finanziellen Einnahmen (Grundeinkommen, Erwerbsarbeit u.a.) und Konsumsteuern. Das dafür einzusetzende Geldvolumen bemisst sich aus der Geldumlaufgeschwindigkeit, dem gesellschaftlichen Bedarf sowie dem realen und potentiellen Leistungsangebot. ${ }^{10}$

In den Diskussionen zum Grundeinkommen werden die unmittelbaren und mittelbaren Folgen seiner Einführung unsystematisch antizipiert. Zu einer umfassenden Konzeption eines Systems „Grundeinkommen“ gehören nicht nur Geldverfügbarkeit, soziale Einbindung und Sinnstiftung der eigenen Existenz, sondern auch die Möglichkeit, den existentiellen Arbeitszwang entschärfen zu können (Kellermann 1979, S. 109 ff.). Dazu bedarf es stets von neuem den gesellschaftlichen Erfordernissen anzupassender Arbeitsorganisationen im Mikro- wie im Makrobereich. Grundeinkommen ist eben nur Grundeinkommen. Um nicht nur persönlich höherem Geldbedarf nachkommen zu können sowie Arbeitsfreude, Selbstorganisation, Kreativität und Produktivität zu ermöglichen, sind auch zum einen verschiedene infrastrukturelle Ausstattungen (Wasser, Strom, Transport-, Kommunikations- und Schulungssysteme) sowie zum anderen Zugänge zu materiellen und immateriellen Produktions- und Kreditmitteln, zu Organisationen und Institutionen erforderlich. ${ }^{11}$

Durch das Medium „Geld“, das ja durch seine Verwendung nicht verschwindet, würde sich das System steuern lassen; auch in Bereichen, die nicht aus individueller Initiative bearbeitet werden, aber gesellschaftlich bearbeitet werden müssen. Wer mehr braucht als das Grundeinkommen, kann mit zusätzlicher Erwerbsarbeit zu weiterer Arbeit motiviert werden. Der Fluss des Mediums ließe sich durch Verbrauchssteuern „steuern“: Nicht, wer wirtschaftliche Leistungen verfügbar macht, wird besteuert, sondern wer diese Leistungen beansprucht - wer mehr verbraucht, muss somit mehr zahlen.

10 Das gilt - rational gedacht - auch für den Zusammenhang von Wirtschafts- und Finanzsystem: Bezüglich eines volkswirtschaftlichen Optimums müsste das „Geldprodukt“" (Geldmenge mal Umlaufgeschwindigkeit) mit der „Leistungssumme“ (reale plus potentielle, ungenutzte aber nutzbare, Leistungen) wirtschaftspolitisch stetig ausgeglichen werden.

11 Da, wie gesagt, beim Denken an Geld gewöhnlich das Denken endet, beschränkt sich auch die dominierende Diskussion um Grundeinkommen auf Geldforderungen und Finanzierbarkeit. 
Die Frage, inwieweit sich die Erwartungen verwirklichen ließen, beziehungsweise sich als illusorisch herausstellten, müsste wiederum durch Forschung und passende Experimente (regional, gruppenspezifisch, befristet ...) beantwortet werden können.

Zu erwarten ist je nach Experiment:

- Älteren Menschen, die das alte System gewohnt sind, würde es schwer fallen, umzudenken und sich anders als nach den erworbenen Vorstellungen zu verhalten.

- Jüngere Menschen, die noch nicht durch Existenzängste in ihrem Denken und Handeln beschränkt sind, wären weniger an Gelderwerb, mehr an befriedigender Arbeit orientiert. $^{12}$

- Aufgeschlossene Menschen würden neugierig verschiedenes überlegen und ausprobieren.

- Es würde allgemein weniger zusätzlich bezahlte Arbeit, mehr unentgeltliche Arbeit geleistet.

- Mehr Menschen könnten sich mit ihrem Arbeitsvermögen bezahlt oder unbezahlt der Milderung kleiner und großer Probleme widmen.

- Es könnten freie Zusammenschlüsse zu Arbeitsteams auf Basis gegenseitiger vertraglicher Verpflichtungen entstehen.

- Es ließe sich wieder jene produktive Muße ohne Angst vor Verarmung gewinnen, die benötigtes Nach- und Vordenken ermöglicht.

- Der Wirtschaftskreislauf von Produktion, Distribution und Konsumtion ließe sich von Seiten der Regierungen je nach gegebener Lage von Bedarf und Leistungsvermögen variieren; Wirtschaftsleistungen könnten durch flexibel gehandhabte Geldpolitik in Angebot und Nachfrage funktional gesteuert werden.

Es geht also nicht nur um die Entwicklung ,intelligenter“ Maschinen; um diese auch für das gesellschaftliche Wohl nutzen zu können, geht es vor allem um eine ,intelligente“ Organisation der gesellschaftlich erforderlichen Arbeit. Durch entsprechende Forschung sind diese zu identifizieren und durch differenzierte Ausschreibungen lassen sich die benötigten Arbeitsleistungen mit Hilfe von Geld mobilisieren, das durch die entstehenden Leistungen gedeckt wird.

Gesellschafts- und wirtschaftspolitisch sollte klar sein, dass derzeit in Entwicklung und Anwendung von Automaten mehr an Geist und Geld investiert wird als in Abschätzung und Steuerung der Folgen. Und eben so müsste verstanden werden, dass es Aufgabe der Regierungen und nicht der einzelnen Betriebe und schon gar nicht von Privatpersonen ist, in aufeinander abgestimmten Programmen den gesellschaftlich unerwünschten Folgen von Automation, Digi-

12 Analog zu dem Umstand, dass auf dem Gebiet der USA geborene Kinder die Staatsangehörigkeit erhalten, ließe sich die allmähliche und damit überschaubare Einführung eines Grundeinkommens für Kinder bewerkstelligen: Sie erhalten ab einem noch zu bestimmenden Alter staatlich garantierte Geldverfügbarkeit. 
talisierung und vermehrtem Robotereinsatz zu begegnen, beziehungsweise die Entwicklungen im Interesse des Allgemeinwohls zu steuern.

\section{Abschließende Bemerkung: Arbeitsideologie und Grundeinkommen}

Die vermutlich erfolgreichste Ideologie ${ }^{13}$ der Industriegesellschaft ist die Arbeitsideologie (Kellermann 2002, S. 200 ff.). Ideologie ist eine Weltanschauung und eine Lehre, die das vorherrschende Menschen- und Gesellschaftsbild vorgeben. In einer Ideologie vermengen sich Wahrheit und Verlogenheit zu einer schwierig zu analysierenden Einheit.

Wahr an Arbeitsideologie ist anthropologisch, dass Arbeit zur Sicherung und Verbesserung der menschlichen Lebensbedingungen geleistet werden muss. Die Verlogenheit hängt mit dem zusammen, was seit dem 19. Jahrhundert als „Recht auf Arbeit“ gefordert wird. Weder Leibeigene noch Sklaven und Sklavinnen, Knechte und Mägde oder Dienstmädchen und Lakaien haben in den vergangenen Gesellschaftsverhältnissen ein Recht auf Arbeit gefordert. Diese Forderung entstand mit dem Aufkommen industrieller Produktion.

Die sich stark differenzierende Arbeitsteilung und das Entstehen von Fabriken verursachten den unabdingbaren Tausch von Gütern und Diensten, da eine Selbstversorgung immer schwieriger wurde. Das funktionale Tauschmedium „Geld“ setzte sich im gleichen Maße wie die Spezialisierung und die Ausweitung der Lohnarbeit (Lohn nach Zeit) durch: Das „Beschäftigungssystem“ "entstand. Mit diesem Prozess wurde Geldverfügbarkeit immer dringlicher, was die „Geldgesellschaft“ begründete.

Zur Arbeitsideologie gehört, dass Geld legal und legitim vor allem durch Arbeit zu erwerben ist und dabei nur Erwerbsarbeit als Arbeit verstanden wird. Es besteht folglich ein sozialer Zwang zu Erwerbsarbeit. Verlogen ist diese Ideologie, weil unter „Recht auf Arbeit“ eigentlich lediglich ein Recht auf Erwerbsarbeit, auf „Beschäftigung“, auf einen „Arbeitsplatz“verstanden wird. ${ }^{14}$ Genauer: Es ist ein Recht auf Einkommen gemeint, da fast alle zum Leben erforderlichen Güter und Dienste gekauft werden müssen. In Wahrheit geht es also letztlich um das „Recht auf Leben“ in der Geldgesellschaft. Die durch Technik und Arbeitsteilung entwickelten Spezialisierungen und Produktivitätssteigerungen des begonnenen Digitalen Zeitalters ermöglichen jedoch einen Epochenwechsel in der gesellschaftlichen Organisation der erforderlichen Arbeit

13 Altgriech: $\varepsilon \tilde{i} \delta o \varsigma$ das Bild, die äußere Erscheinung und $\lambda o ́ \gamma o \varsigma$ die Rede, Erzählung (Menge 1903, S. 167 bzw. 350 f.)

14 Die individuelle Unfähigkeit, Zusammenhänge zu erkennen und zu benennen, bzw. die politische Verlogenheit geht so weit, dass nicht nur im deutschen Bundestagswahlkampf 2013 Slogans der folgenden Art plakatiert wurden: „Damit Europa mehr Arbeit und Wachstum schafft“ (CDU) oder „Frieden sichern, Millionäre besteuern [...] Beschäftigung schaffen [...]“ (Die Linke). Im eigentlichen Wortsinn muss Arbeit nicht geschaffen, sondern geleistet werden; und Leute müssen nicht beschäftigt werden. Es geht um Arbeit zur Sicherung und Verbesserung der gesellschaftlichen Lebensverhältnisse. 
und damit eine Entschleierung der Arbeitsideologie: eine relative Lockerung des kollektiven und eine Befreiung des individuellen Arbeitszwangs durch ein funktional begründetes „Recht auf Grundeinkommen“".

Wenn das Angebot produzierter Güter und verfügbarer Dienste wegen strukturell sehr ungleicher Kaufkraft nicht nachgefragt werden kann, ist das gesamtgesellschaftlich dysfunktional, weil das Wirtschaftssystem stagniert oder gar kollabiert. Gesellschaftlich funktional hingegen ist die Vermittlung von Produktion, Distribution und Konsumtion des Wirtschaftssystems durch „mobiles“, also umlaufendes, Geld: Roboter konsumieren nicht, sehr wohl aber Menschen mit Bedürfnissen, die wenigstens ein Grundeinkommen haben. Damit kann der mit Geld vermittelte wirtschaftliche „Kreislauf“ in Gang gehalten werden und den Menschen wird die Wahl zwischen unentgeltlicher Tätigkeit, selbständiger oder unselbständiger Erwerbsarbeit ermöglicht.

Wie müssen sich unsere immer noch weitgehend starren und ausgeprägt hierarchischen Organisationsformen in den Unternehmen wandeln, damit sich das Potenzial der Beschäftigten in selbstorganisierten, agilen Innovationsprozessen über Abteilungs- und Disziplingrenzen hinweg entfalten kann? (Pfeiffer, Suphan 2015, S. 30)

Auf der Basis von Künstlicher Intelligenz, Robotern und digitalisierter Vernetzung ließe sich nach den physisch oder finanziell erzwungenen Arbeitsverhältnissen in Sklaven-, Lohnarbeits- und Geldgesellschaften eine neue gesellschaftliche Organisation der Arbeit für ein wirtschaftlich gesichertes, freieres Arbeitssystem mit Einkommensgarantie entwickeln. Rationalfunktionale Argumente für garantiertes Einkommen sind zum einen: Wenn privatwirtschaftlich in Automaten investiert wird, um auf Märkten deren Produkte absetzen zu können, muss die gewünschte Nachfrage mit Geld ausgestattet sein. Nochmals: Nachfrage kann nur dann entstehen, wenn beides, Bedarf und Geldverfügbarkeit, gegeben sind. Zum anderen: Arbeitszwang ist, gleichgültig in welcher Gesellschaft und auf welchen Gebieten, wirtschaftlich weniger effizient als intrinsisch motivierte Tätigkeit. ${ }^{15}$

\section{Anhang}

Zitate zur Einstimmung in die Problematik aus „Der Maschinen-Schwarm“ von Alexander Jung und Thomas Schulz (2015):

„Iiwa ist ein Roboter der neuen Generation, ein erstaunlich gefühlvolles Geschöpf.“ (S. 70)

„Roboter werden immer schneller, kleiner und klüger.“ (S. 71)

„Roboter nehmen Chirurgen mühselige Aufgaben wie das Entfernen von Gewebe ab.“ (S. 71)

15 Das gilt auch für das Lernen in Schule und Hochschule: Mit dem heteronomen Studium in Folge des europaweiten Bologna-Prozesses an Universitäten beispielsweise war das Ziel des Europäischen Rats (Lissabon 23./24. März 2000) ,, to become the most competitive and dynamic knowledge based economy of the world" nicht erreichbar. 
„Es (Boston Dynamics, P.K.) hat neben vierbeinigen Robotern, die bis zu 46 Kilometer pro Stunde schnell rennen können, auch humanoide, zweibeinig laufende Maschinen entwickelt.“ (S. 72)

„Sie (die Roboter, P.K.) erkennen sich gegenseitig, tauschen sich aus, lernen und erarbeiten gemeinsam Lösungen. Dann findet der eine Roboter einen Defekt, und der andere repariert ihn.“ (S. 72)

„Im kommenden Jahrzehnt werde der Anteil der Automatisierung in der Fertigung von jetzt 10 auf dann 25 Prozent steigen, prognostiziert die Beratungsgesellschaft Boston Consulting Group (BCG) in einer neuen Studie. Denn die neuen Roboter und intelligenten Maschinen sind nicht nur besser, sondern werden auch immer billiger." (S. 73)

„Schon jetzt kostet ein Roboter in der amerikanischen Elektronikbranche im Schnitt nur vier Dollar die Stunde gegenüber dem Arbeiterlohn von 24 Dollar pro Stunde.“ (S. 73)

„Und auch für die Lohnentwicklung der verbleibenden Arbeiter verheißt die billige Maschinenkonkurrenz nichts Gutes.“ (S. 73)

„Zugleich verspricht die Automatisierung deutlich wachsende Produktivität.“(S. 73)

\section{Literatur}

Brauck, Markus, Alexander Jung, Ann-Kathrin Nezik und Thomas Schulz. 2015. Von A bis Z. In: Der Spiegel Nr. 34/14.8.2015: 9-17.

Europäischer Rat. 2000. Schlussfolgerungen des Vorsitzes, 23. Und 24. März 2000, Lissabon. http://www. europarl.europa.eu/summits/lis1_de.htm, (17.06.2008).

Frey, Carl Benedikt and Michael A. Osborne. 2013. The Future of Employment: How susceptible are jobs to computerisation? Department of Engineering Science, University of Oxford, Oxford, OX1 3PJ, United Kingdom.mosb@robots.ox.ac.uk. Users/michaelosborne/Documents/Research/future_of_employment_paper/old/.texpadtmp/future_of_employment_18.dvi (3.7.2015).

GENESIS-Datenbank. 2015. Erwerbstätige im Inland nach Wirtschaftssektoren, Deutschland. https:// www-genesis.destatis.de/genesis/online (19.2.2016).

Hughes, James. 2014. A Strategic Opening for a Basic Income Guarantee in the Global Crisis. In: Journal of Evolution \& Technology, Vol. 24 Issue 1/ February 2014: 45-61.

Jung, Alexander und Thomas Schulz. 2015. Der Maschinen-Schwarm. In: Der Spiegel Nr. 9/21.2.2015: 70-73.

Kellermann, Paul. 1979. Soziologische Aspekte der Arbeitsmarktpolitik. In: Die außerökonomischen Aspekte der Arbeitsmarktpolitik, hrsg. Ernst Gehmacher: 91-113. Wien: IFES. Nachdruck in Technologie und Politik, Die Zukunft der Arbeit 3, hrsg. Freimut Duve. 1980. 146-181. Reinbek bei Hamburg: Rowohlt Taschenbuchverlag.

Kellermann, Paul. 1981. Arbeit und Bildung II. Klagenfurt: Kärntner Druck- und Verlagsanstalt. 
Kellermann, Paul. 2002. Soziologie und globale Herausforderungen: Zum Verhältnis von Bedürfnis, Arbeit und Ware. In: Kärntner Jahrbuch für Politik 20, hrsg. Karl Anderwald, Peter Karpf und Hellwig Valentin, 189-212. Klagenfurt: Kärntner Druck- und Verlagsanstalt.

Kellermann, Paul. 2007. Moneyismus - Der Glaube an Geld als Alltagsreligion. In: Die Geldgesellschaft und ihr Glaube, hrsg. Paul Kellermann, 115-125. Wiesbaden: VS Verlag für Sozialwissenschaften.

Kellermann, Paul. 2014a. Soziologie des Geldes. Wiesbaden: Springer VS.

Kellermann, Paul. 2014b. Zum Verhältnis von Bedürfnis, Arbeit und Geld. In: Soziologie des Wirtschaftlichen, hrsg. Dieter Bögenhold, 239-265. Wiesbaden: Springer VS.

Löger, Christoph. 2016. 208 Sekunden. In: auto touring feb/16, 20-22. Wien: ÖAMTC-Verlag.

Menge, Hermann. 1903. Griechisch-Deutsches Schulwörterbuch. Berlin-Schöneberg: Langenscheidtsche Verlagsbuchhandlung.

Pfeiffer, Sabine und Anne Suphan. 2015. Der AV-Index. Lebendiges Arbeitsvermögen und Erfahrung als Ressourcen auf dem Weg zu Industrie 4.0. Working Paper 2015 \#1 (draft v1.0 vom 13.04.2015), Universität Hohenheim.

Samuelson, Paul A. und William D. Nordhaus. 2005. Volkswirtschaftslehre. Landsberg am Lech: mi-Fachverlag.

Sobotka, Tomás, Krystof Zeman, Michaela Potancokova, Jakob Eder, Zuzanna Brzozowska, Éva Beaujouan and Anna, Matysiak. 2015. European Fertility Datasheet 2015. Wien: Vienna Institute of Demography.

Union of International Associations (1907-2015): The Encyclopedia of World Problems and Human Potential. http://www.uia.org/encyclopedia (17.2.2016).

Urschitz, Josef. 2015. Die Gelddrucker sind kläglich gescheitert. In: Die Presse 21.8.2015: 15.

Paul Kellermann, geb. 1937, deutsche und österr. Staatsbürgerschaft, Diplom in Soziologie Frankfurt 1963, Dr. oec. publ. 1966 München, Venia legendi Allgemeine Soziologie 1972 Konstanz, o. Prof. 1973 Klagenfurt, Gastaufenthalte an Universitäten in Deutschland, Österreich, USA, Äthiopien, Chile, Tansania. Associated Prof. University of Northern Iowa, em. Univ. Prof. Universität Klagenfurt. 